\title{
IMPROVED PRECISION OF FIXED-POINT ALGORITHMS BY MEANS OF COMMON FACTORS
}

\author{
Yuriy A. Reznik* \\ Information Systems Laboratory \\ Stanford University \\ 353 Serra Mall, Stanford, CA 94305 \\ Email: yreznik@stanford.edu
}

Arianne T. Hinds, and Joan L. Mitchell

\author{
Ricoh | IBM \\ InfoPrint Solutions Company \\ 6300 Diagonal Hwy, Boulder, CO, 80301 \\ Email: $\{$ arianne, joanm $\} @$ us.ibm.com
}

\begin{abstract}
We describe a general techniques for improving precision of fixed-point implementations of signal processing algorithms (such as filters, transforms, etc.) by introducing "common factors". These factors are applied to groups of real constants that need to be approximated by dyadic rational numbers, and we show that by carefully choosing values of common factors, the errors of final dyadic rational approximations can be significantly reduced. We show that the problem of design of such approximations is related to the classic Diophantine approximation problem, and include examples explaining how it can be solved, and used for improving practical designs.
\end{abstract}

Index Terms - Signal processing, fixed point algorithms, Diophantine approximations.

\section{INTRODUCTION}

One of the most basic tasks that arises in the design of fixedpoint signal processing algorithms is that of approximating a given set of real (and possibly irrational) constants $\theta_{1}, \ldots, \theta_{m}$, $(m \geqslant 2)$ with a set of rational numbers with common dyadic denominator:

$$
\theta_{1} \approx p_{1} / 2^{k}, \ldots, \theta_{m} \approx p_{m} / 2^{k},
$$

where $p_{1}, \ldots, p_{m}$, and $k$ are integers.

This way the numbers $\theta_{1}, \ldots, \theta_{m}$ can be approximately represented in computer's memory by integers $p_{1}, \ldots, p_{m}$, which, in turn, can be used for execution of basic arithmetic operations. For example, multiplications of an input variable $x$ by $\theta_{1}, \ldots, \theta_{m}$ can be conveniently mapped into integer instructions as follows:

$$
x \theta_{i} \approx x p_{i} / 2^{k} \rightsquigarrow\left(x * p_{i}\right) \gg k, \quad(i=1, \ldots, m)
$$

where $*$ and $\gg$ denote integer multiplication and bit-wise right shift operations correspondingly.

\footnotetext{
* On leave from Qualcomm Inc., 5775 Morehouse Dr., San Diego, CA.
}

The key parameter that influences the complexity of algorithms using dyadic rational approximations (1) is the number of "precision bits" $k$. In software designs, this parameter is often constrained by the width of registers (e.g. 8, 16 or 32 ), and failure to meet such a constraint can possibly result in doubling (or in some cases - quadrupling) of the execution time. In hardware designs parameter $k$ directly affects the number of gates needed to implement adders and multipliers.

The precision of approximations (1) also depends on the parameter $k$. Thus, given $k$ and $\theta_{i}$, the best choice of $p_{i}$ yields $\left|\theta_{i}-p_{i} / 2^{k}\right|=2^{-k}\left|2^{k} \theta_{i}-p_{i}\right|=2^{-k} \min _{z \in \mathbb{Z}}\left|2^{k} \theta_{i}-z\right| \leqslant 2^{-k-1}$, which means, that minimum worst case magnitude of error

$$
\Delta(k)=\min _{p_{1}, \ldots, p_{m}} \max _{i}\left\{\left|\theta_{i}-p_{i} / 2^{k}\right|\right\} .
$$

is also bounded by

$$
\Delta(k) \leqslant 2^{-k-1} .
$$

In simple terms, this means, that on average, each bit of precision in dyadic approximations (1) reduces their worst case error at least by half.

This last observation is crucial for understanding precision-complexity tradeoffs in conventional fixed-point designs. It also underscores the importance of finding more efficient (with faster decaying errors) techniques for "importing" of real (and in particular, irrational) numbers into fixed-point algorithms.

In this paper, we study one such possible technique, involving the use of a "common factor". The main idea of this technique is to introduce an additional parameter $\xi$ and use it for minimization of errors in approximations:

$$
\theta_{1} \xi \approx p_{1} / 2^{k}, \ldots, \theta_{m} \xi \approx p_{m} / 2^{k} .
$$

where $p_{1}, \ldots, p_{m}$, and $k$ are integers.

We note, that in many practical situations, the uniform scale of the original values $\theta_{1}, \ldots, \theta_{m}$ by $\xi$ can be either ignored (e.g. when it has no effect on the output), or "neutralized" by applying the inverse factor $1 / \xi$ to constants in adjacent stages of the algorithm. In other words, we assume that 
the use of our modified approximations (4) instead of direct ones (1) will be feasible in practical designs.

We show, that for infinitely many $k$, by carefully choosing the value of a common factor $\xi$ the equivalent (scaled by $1 / \xi$ ) worst case error of approximations (4):

$$
\Delta_{\xi}(k)=\frac{1}{\xi} \min _{p_{1}, \ldots, p_{m}} \max _{i}\left\{\left|\theta_{i} \xi-p_{i} / 2^{k}\right|\right\}
$$

can be made as small as

$$
\Delta_{\xi}(k) \lesssim 2^{-k\left(1+\frac{1}{m-1}\right)} .
$$

In other words, we show that common-factor-based approximations can be significantly more precise than direct ones. We note that the magnitude of the achievable gain is particularly striking for small $m$. For example, when $m=2$, the right side in (6) turns into $2^{-2 k}$, which implies, that the use of a common-factor might reduce the number of required precision bits by half!

The rest of this paper is organized as follows. Section 2 contains analysis of common-factor-based approximations and formulation of our main results. Practical examples of using this technique are given in Section 3.

\section{PRECISION OF APPROXIMATIONS WITH COMMON FACTORS}

\subsection{Minimizing errors of pairs of approximations.}

Consider first a special case when $m=2$. By $\delta_{1}(\xi)$ and $\delta_{2}(\xi)$ we denote individual errors of approximations (4):

$$
\delta_{1}(\xi)=\theta_{1} \xi-p_{1} / 2^{k}, \quad \delta_{2}(\xi)=\theta_{2} \xi-p_{2} / 2^{k},
$$

and our first task would be to see if $\max \left\{\left|\delta_{1}(\xi)\right|,\left|\delta_{2}(\xi)\right|\right\}$ can be minimized by adjusting $\xi$.

We claim the following.

Lemma 1. Let $\theta_{1}, \theta_{2}$ be real numbers, such that $\theta_{1} \theta_{2}>0$, and let $k, p_{1}$, and $p_{2}$ be integers. Then, there exist values $\xi^{*}$ and $\delta^{*}$, such that

$\delta^{*}=\max \left\{\left|\delta_{1}\left(\xi^{*}\right)\right|,\left|\delta_{2}\left(\xi^{*}\right)\right|\right\}=\min _{\xi} \max \left\{\left|\delta_{1}(\xi)\right|,\left|\delta_{2}(\xi)\right|\right\}$

These values are:

$$
\xi^{*}=\frac{1}{2^{k}} \frac{p_{1}+p_{2}}{\theta_{1}+\theta_{2}}
$$

and

$$
\delta^{*}=\frac{1}{2^{k}}\left|\theta_{1} \frac{p_{1}+p_{2}}{\theta_{1}+\theta_{2}}-p_{1}\right|=\frac{1}{2^{k}}\left|\theta_{2} \frac{p_{1}+p_{2}}{\theta_{1}+\theta_{2}}-p_{2}\right| .
$$

Proof. Condition $\theta_{1} \theta_{2}>0$ implies that both $\delta_{1}(\xi)$ and $\delta_{2}(\xi)$ are non-constant and have the same direction of growth with $\xi$.

If $\delta_{1}(\xi)$ and $\delta_{2}(\xi)$ intersect 0 at the same location, then there exists point $\xi^{*}$ such that $\delta_{1}\left(\xi^{*}\right)=\delta_{2}\left(\xi^{*}\right)=0$. This implies that

$$
\xi^{*}=\frac{1}{2^{k}} \frac{p_{1}}{\theta_{1}}=\frac{1}{2^{k}} \frac{p_{2}}{\theta_{2}}
$$

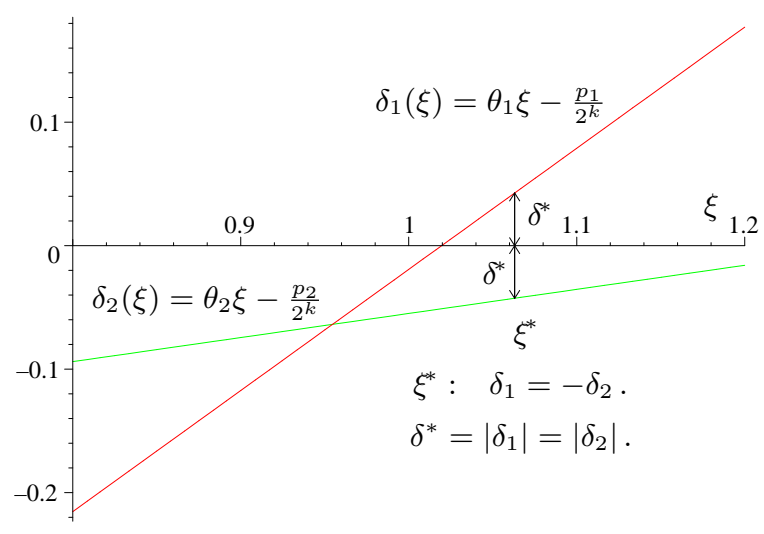

Fig. 1. Finding $\min _{\xi} \max \left\{\left|\theta_{1} \xi-\frac{p_{1}}{2^{k}}\right|,\left|\theta_{2} \xi-\frac{p_{2}}{2^{k}}\right|\right\}$.

which is a special case of (8).

If $\delta_{1}(\xi)$ and $\delta_{2}(\xi)$ intersect 0 at different locations, then there exists $\xi^{*}$ such that (see Fig. 1):

$$
\delta_{1}\left(\xi^{*}\right)=-\delta_{2}\left(\xi^{*}\right)
$$

Moreover, since both $\delta_{1}(\xi)$ and $\delta_{2}(\xi)$ have same direction of growth, moving $\xi$ away from $\xi^{*}$ will lead to asymmetric changes in absolute values of $\delta_{1}(\xi)$ or $\delta_{2}(\xi)$. That is, one of them will increase. Therefore, $\xi^{*}$ is the point of minimum of $\max \left\{\left|\delta_{1}(\xi)\right|,\left|\delta_{2}(\xi)\right|\right\}$.

By solving (10) with respect to $\xi^{*}$ we arrive at formula (8), and by plugging (8) in (7), and using (10) we arrive at (9).

\subsection{Associated Diophantine approximation}

Let us now further assume that $p_{1}, p_{2}$ have same signs as $\theta_{1}$, and $\theta_{2}$. Then, by denoting $p=p_{1}, q=p_{1}+p_{2}$, and

$$
\theta^{*}=\frac{\theta_{1}}{\theta_{1}+\theta_{2}}
$$

we observe that both parts of (9) turn into

$$
\delta^{*}=\frac{|q|}{2^{k}}\left|\theta^{*}-p / q\right| .
$$

By further de-scaling this quantity by $\xi^{*}$ we arrive at

$$
\delta^{*} / \xi^{*}=\left|\theta_{1}+\theta_{2}\right|\left|\theta^{*}-p / q\right|,
$$

which means, that by plugging $\xi=\xi^{*}$, the problem of finding minimum of the worst case error of a pair of scaled dyadic rational approximations

$$
\Delta_{\xi}(k)=\frac{1}{\xi} \min _{p_{1}, p_{2}} \max \left\{\left|\delta_{1}(\xi)\right|,\left|\delta_{2}(\xi)\right|\right\} .
$$

becomes equivalent to the problem of finding rational approximations of a single number $\theta^{*}$

$$
\theta^{*} \approx p / q
$$


Furthermore, if $\theta^{*}$ is irrational, then (13) turns into a classic Diophantine approximation problem [1].

The following result from Diophantine approximation theory (cf. [1, p. 11, Theorem V]) will be useful in our context.

Fact 1. Let $\theta$ be irrational. Then there exist infinitely many integers $q$ and $p$ such that

$$
|\theta-p / q|<\kappa(\theta) q^{-2}
$$

where:

$$
\kappa(\theta)=\left[\begin{array}{ll}
5^{-1 / 2}, & \text { if } \theta=\frac{r \psi+s}{u \psi+v}, \text { where: } \\
& \psi=\frac{\sqrt{5}-1}{2} ; r, s, u, v \in \mathbb{Z}, \\
& \text { such that } r v-u s= \pm 1 \\
2^{-3 / 2}, & \text { otherwise. }
\end{array}\right.
$$

\subsection{Main result for approximations of pairs of constants}

We state the following.

Theorem 1. Let $\theta_{1}, \theta_{2}$ be irrational numbers of the same sign. Then, there exist infinitely many integers $k$ and real numbers $\xi$, such that

$$
\begin{aligned}
\Delta_{\xi}(k) & =\frac{1}{\xi} \min _{p_{1}, p_{2}} \max \left\{\left|\theta_{1} \xi-p_{1} / 2^{k}\right|,\left|\theta_{2} \xi-p_{2} / 2^{k}\right|\right\} \\
& <\kappa\left(\frac{\theta_{1}}{\theta_{1}+\theta_{2}}\right) \frac{4}{\left|\theta_{1}+\theta_{2}\right|} 2^{-2 k}=O\left(2^{-2 k}\right)
\end{aligned}
$$

Proof. We use the following construction.

By assuming that $\xi=\xi^{*}$, and solving the associated Diophantine approximation problem (13), we find integers $p, q$ satisfying precision constraint (14) of Fact 1. This also gives us integer factors $p_{1}=p$ and $p_{2}=q-p$ for our dyadic approximations. In order to select $k$, we can use some additional constraints. For example, we can require

$$
1 / 2<\xi^{*} \leqslant 1
$$

which is satisfied by choosing $k=\left\lceil\log _{2}\left(q /\left(\theta_{1}+\theta_{2}\right)\right)\right\rceil$.

Then, by plugging Diophantine precision bound (14) in (12), using lower bound for $\xi^{*}$ from (17), and some simple algebra, we arrive at expression (16) claimed by the theorem.

\subsection{Extension of analysis to m-ary case}

We now turn our attention to a problem of finding dyadic rational approximations for larger $(m>2)$ sets of numbers:

$$
\theta_{1} \xi \approx p_{1} / 2^{k}, \ldots, \theta_{m} \xi \approx p_{m} / 2^{k}
$$

For simplicity, we assume that all numbers $\theta_{1}, \ldots, \theta_{m}$ and $p_{1}, \ldots, p_{m}$ are either positive or negative.

From Lemma 1, we know that for any pair of numbers $\theta_{i}, \theta_{j}, i \neq j$, we can compute factor

$$
\xi_{i j}^{*}=\frac{1}{2^{k}} \frac{p_{i}+p_{j}}{\theta_{i}+\theta_{j}},
$$

which will "symmetrize" errors of approximations:

$$
\delta_{i j}^{*}=\frac{1}{2^{k}}\left|\theta_{i} \frac{p_{i}+p_{j}}{\theta_{i}+\theta_{j}}-p_{i}\right|=\frac{1}{2^{k}}\left|\theta_{j} \frac{p_{i}+p_{j}}{\theta_{i}+\theta_{j}}-p_{j}\right| .
$$

and which will turn them into a Diophantine approximation:

$$
\delta_{i j}^{*}=\frac{\left|q_{i j}\right|}{2^{k}}\left|\theta_{i j}^{*}-p_{i j} / q_{i j}\right| .
$$

where $p_{i j}=p_{i}, q_{i j}=p_{i}+p_{j}$, and

$$
\theta_{i j}^{*}=\frac{\theta_{i}}{\theta_{i}+\theta_{j}} .
$$

By applying $\xi_{i j}^{*}$ to the remaining constants $\left\{\theta_{k}, k \neq i, j\right\}$, we note that their approximations also turn into Diophantines $\left|\theta_{k} \xi_{i j}^{*}-p_{k} / 2^{k}\right|=\frac{1}{2^{k}}\left|\theta_{k} \frac{p_{i}+p_{j}}{\theta_{i}+\theta_{j}}-p_{k}\right|=\frac{\left|q_{i j}\right|}{2^{k}}\left|\theta_{k \mid i j}^{*}-p_{k} / q_{i j}\right|$, where, however, the resulting constants

$$
\theta_{k \mid i j}^{*}=\frac{\theta_{k}}{\theta_{i}+\theta_{j}}
$$

and errors of their approximations are different.

This means that by using factor $\xi_{i j}^{*}$ we can reduce the problem of finding $m$ dyadic rational approximations (18) to one of finding $m-1$ simultaneous Diophantine approximations:

$$
\theta_{i j}^{*} \approx p_{i j} / q_{i j},\left\{\theta_{k \mid i j}^{*} \approx p_{k} / q_{i j}, k \neq i, j\right\} .
$$

The relevant result from Diophantine approximation theory is given below (cf. [1, p. 14, Theorem III], [2, p.138]):

Fact 2. Let $\theta_{1}, \ldots, \theta_{m},(m \geqslant 2)$ be irrationals. Then, there are infinitely many integers $q$ and $p_{1}, \ldots, p_{m}$, such that

$$
\max _{i}\left\{\left|\theta_{i}-p_{i} / q\right|\right\}<\frac{m}{m+1} q^{-1-1 / m} .
$$

We are now ready to formulate and prove our main result.

Theorem 2. Let $\theta_{1}, \ldots, \theta_{2}$ be $m>2$ irrational numbers of the same sign. Then, there exist infinitely many integers $k$ and real values $\xi$, such that

$$
\begin{aligned}
\Delta_{\xi}(k) & =\frac{1}{\xi} \min _{p_{1}, \ldots, p_{m}} \max _{i}\left\{\left|\theta_{i} \xi-p_{i} / 2^{k}\right|\right\} \\
& <\frac{m-1}{m}\left(\min _{i j}\left\{\left|\theta_{i}+\theta_{j}\right|\right\}\right)^{-\frac{1}{m-1}} 2^{-(k-1)\left(1+\frac{1}{m-1}\right)} \\
& =O\left(2^{-k\left(1+\frac{1}{m-1}\right)}\right) .
\end{aligned}
$$

Proof. We use the following construction.

We scan all $\left(\begin{array}{c}m \\ 2\end{array}\right)$ pairs of indices $i, j$, and find a pair, for which the normalized (by $1 / \xi_{i j}^{*}$ ) worst case error:

$$
\begin{aligned}
& \frac{1}{\xi_{i j}^{*}} \min _{p_{i j}, p_{k}} \frac{\left|q_{i j}\right|}{2^{k}} \max \left\{\left|\theta_{i j}^{*}-\frac{p_{i j}}{q_{i j}}\right|,\left|\theta_{k \mid i j}^{*}-\frac{p_{k}}{q_{i j}}\right|, k \neq i, j\right\} \\
& \quad=\left|\theta_{i}+\theta_{j}\right| \min _{p_{i j}, p_{k}} \max \left\{\left|\theta_{i j}^{*}-\frac{p_{i j}}{q_{i j}}\right|,\left|\theta_{k \mid i j}^{*}-\frac{p_{k}}{q_{i j}}\right|, k \neq i, j\right\}
\end{aligned}
$$

is the smallest one.

Then, by applying Fact 2, using (19) to replace $q_{i j}$ with $2^{k}$ and $\xi_{i j}^{*}$, and subsequently, bounds $1 / 2<\xi_{i j}^{*} \leqslant 1$ (which is attainable by choice of $\mathrm{k}$ ), and $\left|\theta_{i}+\theta_{j}\right| \geqslant \min _{i j}\left\{\left|\theta_{i}+\theta_{j}\right|\right\}$, we arrive at estimate (26) claimed by the theorem. 
Table 1. Approximations of a pair of constants $\theta_{1}=\cos \left(\frac{\pi}{16}\right)$, and $\theta_{2}=\cos \left(\frac{7 \pi}{16}\right)$.

\begin{tabular}{|c|c|c|c|c|c|c|c|c|c|c|}
\hline \multicolumn{4}{|c|}{$\begin{array}{l}\text { Direct dyadic approximations: } \\
\theta_{1} \approx p_{1} / 2^{k}, \quad \theta_{2} \approx p_{2} / 2^{k}\end{array}$} & \multicolumn{3}{|c|}{$\begin{array}{l}\text { Associated Diophantine approximation: } \\
\qquad \theta^{*}=\theta_{1} /\left(\theta_{1}+\theta_{2}\right) \approx p / q\end{array}$} & \multicolumn{4}{|c|}{$\begin{array}{l}\text { Dyadic approximations with common factor } \xi^{*} \text { : } \\
\qquad \theta_{1} \xi^{*} \approx p_{1} / 2^{k}, \quad \theta_{2} \xi^{*} \approx p_{2} / 2^{k}\end{array}$} \\
\hline$k$ & $p_{1}$ & $p_{2}$ & $\max _{i}\left|\theta_{i}-p_{i} / 2^{k}\right|$ & $q$ & $p$ & $\left|\theta^{*}-p / q\right|$ & $\xi^{*}=\frac{1}{2^{k}} \frac{q}{\theta_{1}+\theta_{2}}$ & $p_{1}$ & $p_{2}$ & $\frac{1}{\xi^{*}} \max _{i}\left|\theta_{i} \xi^{*}-p_{i} / 2^{k}\right|$ \\
\hline 1 & 2 & 0 & 0.1950903220 & & & & & & & \\
\hline 2 & 4 & 1 & 0.0549096780 & & & & & & & \\
\hline 3 & 8 & 2 & 0.0549096780 & 6 & 5 & 0.0007559856 & 0.6378225711 & 5 & 1 & 0.0008889451 \\
\hline 4 & 16 & 3 & 0.0192147196 & & & & & & & \\
\hline 5 & 31 & 6 & 0.0120352804 & & & & & & & \\
\hline 6 & 63 & 12 & 0.0075903220 & & & & & & & \\
\hline 7 & 126 & 25 & 0.0035897196 & & & & & & & \\
\hline 8 & 251 & 50 & 0.0003165304 & & & & & & & \\
\hline 9 & 502 & 100 & 0.0003165304 & 440 & 367 & 0.0000015901 & 0.7308383627 & 367 & 73 & 0.0000018698 \\
\hline 10 & 1004 & 200 & 0.0003165304 & & & & & & & \\
\hline 11 & 2009 & 400 & 0.0002221780 & 1543 & 1287 & 0.0000001172 & 0.6407293146 & 1287 & 256 & 0.0000001378 \\
\hline
\end{tabular}

Table 2. Approximations of a pair of constants $\theta_{1}=\cos \left(\frac{3 \pi}{16}\right)$, and $\theta_{2}=\cos \left(\frac{5 \pi}{16}\right)$.

\begin{tabular}{|c|c|c|c|c|c|c|c|c|c|c|}
\hline \multicolumn{4}{|c|}{$\begin{array}{l}\text { Direct dyadic approximations: } \\
\theta_{1} \approx p_{1} / 2^{k}, \quad \theta_{2} \approx p_{2} / 2^{k}\end{array}$} & \multicolumn{3}{|c|}{$\begin{array}{l}\text { Associated Diophantine approximation: } \\
\qquad \theta^{*}=\theta_{1} /\left(\theta_{1}+\theta_{2}\right) \approx p / q\end{array}$} & \multicolumn{4}{|c|}{$\begin{array}{l}\text { Dyadic approximations with common factor } \xi^{*} \text { : } \\
\qquad \theta_{1} \xi^{*} \approx p_{1} / 2^{k}, \quad \theta_{2} \xi^{*} \approx p_{2} / 2^{k}\end{array}$} \\
\hline$k$ & $p_{1}$ & $p_{2}$ & $\max _{i}\left|\theta_{i}-p_{i} / 2^{k}\right|$ & $q$ & $p$ & $\left|\theta^{*}-p / q\right|$ & $\xi^{*}=\frac{1}{2^{k}} \frac{q}{\theta_{1}+\theta_{2}}$ & $p_{1}$ & $p_{2}$ & $\frac{1}{\xi^{*}} \max _{i} \mid \theta_{i} \xi^{*}-p_{i} / 2^{k}$ \\
\hline 1 & 2 & 1 & 0.1685303877 & & & & & & & \\
\hline 2 & 3 & 2 & 0.0814696123 & 5 & 3 & 0.0005438163 & 0.9011997775 & 3 & 2 & 0.0007542949 \\
\hline 3 & 7 & 4 & 0.0555702330 & & & & & & & \\
\hline 4 & 13 & 9 & 0.0189696123 & & & & & & & \\
\hline 5 & 27 & 18 & 0.0122803877 & & & & & & & \\
\hline 6 & 53 & 36 & 0.0069297670 & & & & & & & \\
\hline 7 & 106 & 71 & 0.0033446123 & & & & & & & \\
\hline 8 & 213 & 142 & 0.0008827330 & 367 & 220 & 0.0000011428 & 1.0335634948 & 220 & 147 & 0.0000015851 \\
\hline 9 & 426 & 284 & 0.0008827330 & & & & & & & \\
\hline 10 & 851 & 569 & 0.0004149248 & & & & & & & \\
\hline 11 & 1703 & 1138 & 0.0000938295 & 2207 & 1323 & 0.0000000918 & 0.7769327769 & 1323 & 884 & 0.0000001273 \\
\hline
\end{tabular}

\section{EXAMPLE OF DESIGN USING COMMON FACTORS - BASED APPROXIMATIONS}

As an example, let us consider a design of an $8 \times 8$ Discrete Cosine Transform (DCT) proposed by M. Vetterli and A. Ligtenberg [3]. We notice, that this is a scaled transform (that is, we can move leading factors outside 1D transforms), and that its 1-stage odd butterflies involve multiplications by pairs: $\left(C_{1}, C_{7}\right)$, and $\left(C_{3}, C_{5}\right)$, where $C_{k}=\cos \left(\frac{k \pi}{16}\right)$. Hence, we can introduce common factors for each of these pairs.

We illustrate the process of deriving scaled approximations for these pairs in Tables 1, and 2. First large columns in these tables illustrate the use of direct dyadic rational approximations. It is shown, that their worst case errors decay relatively slowly. Second large columns show solutions for the associated Diophantine approximations. We note that these results are sparse (not every denominator $q$ leads to a precision stated by Fact 1), but their errors decay very rapidly. Finally, in third columns, we show parameters of approximations with common factors, derived from Diophantine solutions.
It can be seen, that common-factor-based approximations are remarkably more precise than the original dyadic rational approximations. For example, the top 3-bit solution from Table 1 , reaches precision which would be normally achievable by using 8 bits. Similar observation can also be made regarding the top (2-bit) common-factor-based solution in Table 2.

In passing, we should note that for some applications precision of those top solutions might already be sufficient. Thus, using these solutions we were able to design an inverse (IDCT) transform passing all compliance tests of the JPEG standard.

\section{REFERENCES}

[1] J. W. S. Cassels, An Introduction to Diophantine Approximations, Cambridge University Press, 1957.

[2] M. Gröetschel, L. Lovácz, and A. Schrijver. Geometric algorithms and combinatorial optimization, Springer, 1988.

[3] M. Vetterli and A. Ligtenberg, "A Discrete Fourier-Cosine Transform Chip", IEEE Journal on Selected Areas in Communications, Vol. SAC-4, No. 1, pp. 49-61, Jan. 1986. 